\title{
Performance Evaluation of 5G Millimeter-Wave based Vehicular Communication for Connected Vehicles
}

This paper was downloaded from TechRxiv (https://www.techrxiv.org).

LICENSE

CC BY 4.0

SUBMISSION DATE / POSTED DATE

$08-12-2021 / 10-12-2021$

\section{CITATION}

Khan, Zadid; Khan, Sakib Mahmud; Chowdhury, Mashrur; rahman, Mizanur; islam, Mhafuzul (2021): Performance Evaluation of $5 \mathrm{G}$ Millimeter-Wave based Vehicular Communication for Connected Vehicles. TechRxiv. Preprint. https://doi.org/10.36227/techrxiv.17139908.v1

$\mathrm{DOI}$ 


\title{
Performance Evaluation of 5G Millimeter- Wave based Vehicular Communication for Connected Vehicles
}

\author{
ZADID KHAN ${ }^{1}$, SAKIB MAHMUD KHAN ${ }^{1}$, Member, IEEE, MASHRUR CHOWDHURY ${ }^{1}$, Senior \\ Member, IEEE, MIZANUR RAHMAN ${ }^{2}$, Member, IEEE, and MHAFUZUL ISLAM ${ }^{3}$ \\ ${ }^{1}$ Glenn Department of Civil Engineering, Clemson University, S Palmetto Boulevard, Clemson, South Carolina 29631, USA \\ ${ }^{2}$ Department of Civil, Construction and Environmental Engineering, 3015 Cyber Hall, Box 870205, Tuscaloosa, Alabama 35487, USA \\ ${ }^{3}$ General Motors, Warren, Michigan, USA \\ Corresponding author: Sakib Mahmud Khan (e-mail: sakibk@clemson.edu).
}

This study is supported by the Center for Connected Multimodal Mobility $\left(\mathrm{C}^{2} \mathrm{M}^{2}\right.$ ) (a US Department of Transportation Tier 1 University Transportation Center) headquartered at Clemson University, Clemson, South Carolina, USA.

\begin{abstract}
Due to the gradual increase in the volume of data generated by connected vehicles (CV), future vehicle-to-infrastructure (V2I) applications will require a communication medium that offers high-speed (high bandwidth) while maintaining reliability in high-mobility traffic scenarios. The $5 \mathrm{G}$ millimeter-wave (mmWave) can solve the communication issues related to V2I applications. However, the performance of the $5 \mathrm{G} \mathrm{mmWave} \mathrm{for} \mathrm{vehicular} \mathrm{communication} \mathrm{in} \mathrm{high-mobility} \mathrm{urban} \mathrm{traffic} \mathrm{scenarios} \mathrm{is} \mathrm{yet} \mathrm{to} \mathrm{be} \mathrm{evaluated.}$ This study presents a case study on assessing the performance of the 5G mmWave based vehicular communication in such traffic scenarios. We have designed three realistic use cases for performance evaluation based on three challenges: increased CV penetration level, dynamic mobility, and V2I application specifications, such as data rate and packet size. Then, we have created a simulation-based experimental setup using a microscopic traffic simulator (SUMO) and a communication network simulator (ns-3) to simulate the use cases. We have used delay, packet loss, throughput, and signal-to-interference-plus-noise ratio (SINR) as the communication performance evaluation metrics. Our analyses found that the $\mathrm{CV}$ penetration level is the primary determinant of the performance of the $5 \mathrm{G} \mathrm{mmWave.} \mathrm{Moreover,} \mathrm{once} \mathrm{the} \mathrm{data} \mathrm{rate} \mathrm{is} \mathrm{increased} \mathrm{by} \mathrm{a}$ factor of 40, delay and packet loss increase by factors of 6.8 and 2.8 , respectively.
\end{abstract}

INDEX TERMS Connected vehicle, 5G, Millimeter-wave communication, Vehicle-to-Infrastructure (V2I)

\section{I.INTRODUCTION}

\section{A. Background}

Connected vehicles (CVs) are an integral part of the transportation cyber-physical system (CPS). Numerous safety, mobility, and environmental benefits can be achieved through CV applications [1]. Several communication technologies are available for vehicular networks, such as the widely adopted Wireless Access in Vehicular Environments (WAVE/IEEE 802.11p), 4G, LTE, Cellular vehicle-to-everything of C-V2X, and $5 \mathrm{G}$ [2]. As identified in previous literature, the advantages of $5 \mathrm{G}$ over $4 \mathrm{G}$ and LTE includes increased spectrum allocation, improved capacity to aggregate simultaneous users within the coverage area, availability of directional beamforming antennas, highly increased bit rates within increased proportions of the $5 \mathrm{G}$ coverage areas, and lower infrastructure cost [3]. Researchers are showing more interest in spectrums higher than $6 \mathrm{GHz}$ to improve communication network reliability and throughput in the transportation CPS
[4], [5]. The frequencies under $6 \mathrm{GHz}$ are already allocated to various LTE bands [6]. The unused spectrums above $6 \mathrm{GHz}$ can provide higher throughput for the increasing number of $\mathrm{CVs}$ in the future. The term 'mmWave' in the ' $5 \mathrm{G}$ mmWave' refers to the spectrum corresponding to wavelengths between 1 and 10 millimeters [7]. Compared to LTE, the carrier frequency of the $5 \mathrm{G}$ mmWave allows for increased data rates while reducing the communication latency [8]. This inherent capacity offered by the $5 \mathrm{G}$ mmWave for both backhaul links (within multiple base stations) and access links (within the base station and end-users) can support the $\mathrm{CV}$ environment [9]-[12]. The coverage area of mmWave communication is limited; however, extending the coverage area through multihop cooperative relay networks can significantly enhance communication network performance [13]. The wireless network operators increase the number of cell towers with reduced cell coverage areas to reduce interference and use cooperative multiple-input-multiple-output (MIMO) antennas at the receiver and sender end to compensate for the reduced 
cell coverage through improved relaying [3]. Future 5G mmWave based deployment will contain a high number of cell towers suitable for the future $\mathrm{CV}$ environment where an unprecedented number of CVs will demand multigigabit/second data transmission to support different $\mathrm{CV}$ applications.

CVs (both human-driven and automated vehicles) can run various data-intensive applications, such as in-vehicle infotainment systems [14] or sensor data sharing [15]. Figure 1 shows a conceptual $5 \mathrm{G}$ mmWave enabled $\mathrm{CV}$ scenario where a data center is connected with the mmWave base station either: (a) directly with a fiber-optic network or (b) via macro base stations. Multiple mmWave base stations can have overlapping regions to serve the high number of $\mathrm{CV}_{\mathrm{s}}$ if needed. The macro base stations operate at lower frequencies (below $6 \mathrm{GHz}$ ) to offer higher wireless communication coverage.

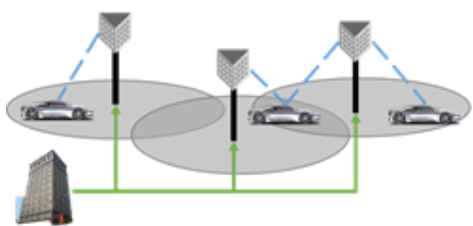

(a)

(b)

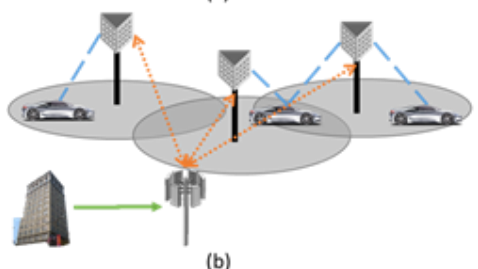

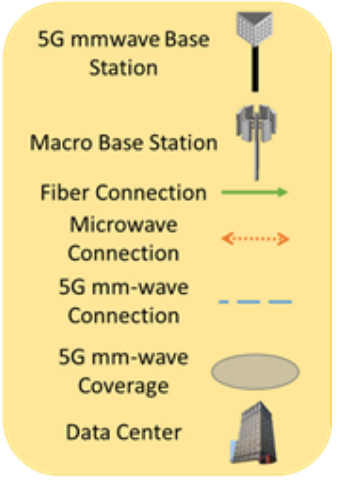

FIGURE 1. $5 G$ mmWave network in urban environment

\section{B. Motivation}

$5 \mathrm{G}$ technologies have been deployed commercially in many locations across the world [16]. However, one of the 5G technologies, the $5 \mathrm{G} \mathrm{mmWave,} \mathrm{is} \mathrm{still} \mathrm{in} \mathrm{the} \mathrm{early} \mathrm{stage} \mathrm{of}$ development and its application feasibility to roadway traffic is an important research area. The performance of the $5 \mathrm{G}$ mmWave base stations and the associated performance metrics for various roadway traffic scenarios is still an evolving research area. The initial phase of deploying any technology should include a thorough evaluation of the technology using simulation studies, identifying potential challenges, and finding the solutions to these challenges. This study was motivated to evaluate the efficacy of $5 \mathrm{G} \mathrm{mmWave}$ in different challenging roadway traffic scenarios in a simulation environment, which can provide valuable data that could be used in the 5G mmWave experiments in a real-world roadway traffic scenario. Our study will provide input to future $5 \mathrm{G}$ mmWave integration in different connected vehicle applications that can be built on the $5 \mathrm{GmmWave}$.

\section{Contributions}

Our contribution in this simulation-based study is providing performance data of $5 \mathrm{G} \mathrm{mmWave} \mathrm{for} \mathrm{V2I} \mathrm{communication} \mathrm{for}$ three different use cases considering three specific challenges: dynamic mobility of $\mathrm{CVs}$, different $\mathrm{CV}$ penetration levels with changing maximum speeds (which is same as the posted speed limit in a corridor), and CV application requirements that correspond to variable data rate and packet size. In the first use case, we evaluate one $5 \mathrm{G} \mathrm{mmWave} \mathrm{base} \mathrm{station's} \mathrm{performance}$ for varying maximum speed of CVs, keeping the other factors (CV penetration level, application data rate, and packet size) constant. To quantify the relationship between CV mobility and the performance of the $5 \mathrm{G} \mathrm{mmWave} \mathrm{communication,} \mathrm{we}$ evaluate the performance of a $5 \mathrm{G}$ mmWave base station with varying numbers of $\mathrm{CVs}$ with the maximum speed of $\mathrm{CVs}$, keeping the other factors (maximum speed, application data rate, and packet size) constant in the second use case. In the third use case, we evaluate one 5G mmWave base station's performance for variable data rate and packet size, keeping the other factors (maximum speed and CV penetration level) constant. We have also selected a baseline technology (WAVE/IEEE 802.11p) for comparison with 5G mmWave. This study will also provide motivation for developing new connected vehicle applications that can benefit from 5G mmWave.

\section{Outline}

In the rest of the paper, we first review the literature on the status of 5G (Subsection II.A) and the use of 5G for vehicular communications (Subsection II.B). Section III describes three use cases developed to evaluate the $5 \mathrm{G}$ mmWave performance. Section IV describes the experimental setup for simulating the use cases. Section VI presents the evaluation results obtained by simulating the use cases. Section VII discusses the conclusions based on the results obtained in Section VI. Section VIII provides recommendations for future research related to the $5 \mathrm{G}$ mmWave based vehicular communication.

\section{LITERATURE REVIEW}

The telecommunications industry and academia have been involved in research to improve spectral communication efficiency and increase the data rate by deploying more LTE base stations. Every ten years, a new generation of emerging communication technologies has been replacing the old technologies since 1980: first-generation analog frequency modulation (FM) cellular systems in 1981, second-generation (2G) digital technology in 1992, 3G in 2001, and LTE-A in 2011 [17]. The 3rd generation partnership project (3GPP) consists of seven standard organizations, and they are responsible for creating the $5 \mathrm{G}$ new radio (NR) standards. They published the first complete set of 5G NR standards in 2017 in Release 15 [18]. Further updates on 5G NR standards will be published in Releases 16 and 17, which will include vehicle-to-everything (V2X) application layer services [19]. 
In the US, the telecommunication industry, including AT\&T, Verizon, and Sprint, have deployed 5G as a fully operational network in major cities, such as Atlanta, Boston, New York, Chicago, San Francisco, and Houston [16].

The barriers to reliable wireless communication for $\mathrm{CV}$ applications include dynamic network topology of vehicular communication due to high vehicle mobility and frequent data link disconnections [20], and cross-channel interference and subsequent packet drop in adjacent channels [21], and higher channel access delay due to an increased number of vehicles [22]. The $5 \mathrm{G}$ is expected to expand and support various use cases, such as enhanced Mobile Broadband (eMBB), UltraReliable Low-Latency Communication (URLLC), and Massive Machine Type Communication (MMTC). eMBB is designed for the high data rate mobile broadband services, which require seamless data access both indoors and outdoors. The URLLC is designed for applications with stringent latency and reliability requirements in highly mobile vehicular communications to enable the CV network. The MMTC supports a vast number of devices that sporadically generate a small amount of data.

Many recent studies have shown that the $5 \mathrm{G}$ mmWave can be applicable for connected vehicles because of its high communication bandwidth with a gigabit/sec data rate and low latency communication delay [9]-[12]. These studies focus on specific aspects of the $5 \mathrm{G}$ mmWave based V2X communication, such as 3D beam alignment strategies [9], software-defined networking-based ecosystem [10], content dissemination methods for enhanced V2X (eV2X) services [11], and propagation channels for vehicle-to-vehicle (V2V) communication [12]. Moreover, end-to-end 5G network slicing can enable mmWave communication for vehicular networks [23]. Dehos et al. have identified mmWave as the primary technology for next-generation communication [24]. Mastrosimone and Panno have studied hybrid mmWave and LTE access links' performance and compared the hybrid system against pure LTE-based access links. They have found CVs can achieve increased throughput of $33 \%$ using the hybrid mmWave and LTE access links compared to the only LTE scenario [25]. Mezzavilla et al. (2018) evaluated LTE and $5 \mathrm{G}$ mmWave communication with a single mobile node [26]. In a real-world experiment conducted by Kim (2019), the author used mmWave for vehicle-to-vehicle communication on a university campus and on city roads with different permitted vehicle speeds. Due to the real-world environment, frequent disconnections occurred, and the inter-vehicle connectivity was impacted by vehicles' speed variations. However, sufficient throughput was maintained to exchange large volume data between the vehicle-to-vehicle (V2V) connectivity. In another study conducted by Giordani et al. (2017), the authors developed a mathematical model to perform connectivity analysis in mmWave-based vehicular networks [27]. Based on their analysis, Giordani et al. (2017) found the mean data throughput at different vehicle speeds remains the same with $5 \mathrm{G}$ mmWave.
As discussed above, no previous study quantified the $5 \mathrm{G}$ mmWave communication performance for different use cases considering dynamic mobility of $\mathrm{CVs}$, different $\mathrm{CV}$ penetration levels with changing maximum speeds, and CV application requirements that correspond to variable data rate and packet size. Our study aims to address these research gaps and provide a simulation-based performance evaluation of the 5G mmWave for V2I applications in an urban roadway corridor. The purpose of our study is to evaluate the $5 \mathrm{G}$ mmWave communication medium that offers high bandwidth while maintaining reliability in high-mobility traffic scenarios. Our multi-vehicle traffic scenario testing is a more realistic extension of an earlier study conducted by Mezzavilla et al. (2018). Also, we demonstrated the comparison of mmWave communication with another widely studied communication option, i.e., WAVE/IEEE 802.11p.

\section{USE CASES AND BASELINE TECHNOLOGY}

Here we discuss the three use cases to evaluate $5 \mathrm{G}$ mmWave communication and the baseline technology, WAVE/IEEE 802.11p whose performance is compared against the $5 \mathrm{G}$ mmWave.

\section{A. USE CASES}

To evaluate the efficacy of the $5 \mathrm{G}$ mmWave, we have selected one urban arterial from Greenville County, South Carolina, US, which is Woodruff Road. Literature suggests that a $5 \mathrm{G}$ mmWave base station can cover a distance of 500m [8], so we have chosen a portion of the corridor $(\sim 500 \mathrm{~m})$ and considered one $5 \mathrm{G}$ mmWave base station. We use a microscopic traffic simulator (i.e., Simulation of Urban Mobility or SUMO) to model the corridor and simulate traffic flow through the corridor. We simulate downlink data traffic, where the remote host sends synthetic data packets with a specific packet size and bit rate, and the CVs receive the data while in motion, which is a V2I application and an eMBB use case of 5G. It is envisioned that one $5 \mathrm{G} \mathrm{mmWave} \mathrm{base} \mathrm{station} \mathrm{can} \mathrm{deliver} 10$ Gbps peak throughput [8], which is very useful when many users are running multiple high data rate applications. Our goal is to study the $5 \mathrm{G} \mathrm{mmWave} \mathrm{communication} \mathrm{performance} \mathrm{for}$ traffic scenarios with multiple CVs on the road network. This study will simulate multiple CVs running applications that need to download data at a high data rate while moving at high speed through the corridor. Figure 2 presents the roadway section we have simulated in this study.

We have mentioned three factors (high $\mathrm{CV}$ penetration level, dynamic mobility, and CV application requirements) that create challenges for the $5 \mathrm{G}$ mmWave-based vehicular communication. Based on these factors, we have designed three use cases to evaluate the performance of the $5 \mathrm{G}$ mmWave. We have also selected a baseline technology for comparison. The description of the three use cases and baseline technology is described here. 


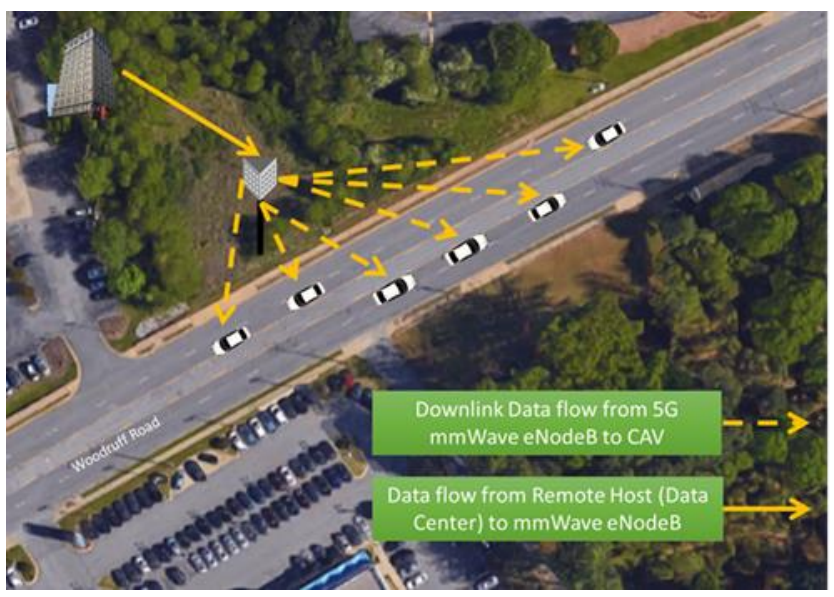

FIGURE 2. CV application data flow (downlink) using $5 \mathrm{G} \mathrm{mmWave} \mathrm{eNB.}$

Use Case 1: CVs represent high-mobility nodes, which will make the communication between CVs and the $5 \mathrm{G}$ mmWave base stations challenging and often unreliable. This study evaluates the effect of $\mathrm{CV}$ mobility on the $5 \mathrm{G} \mathrm{mmWave}$ communication performance for V2I applications. Here, CV mobility refers to the dynamic movement of CVs, which can be expressed using $\mathrm{CV}$ speed. The $5 \mathrm{G}$ mmWave can use any high-frequency spectrum above $24 \mathrm{GHz}$, decreasing communication reliability in high-mobility scenarios. In this use case, we evaluate one 5G mmWave base station's performance for varying maximum speed $(35 \mathrm{mph}, 45 \mathrm{mph}$, $55 \mathrm{mph}$ ) of $\mathrm{CVs}$, keeping the other factors (CV penetration level, application data rate, and packet size) constant. These constant values are: $\mathrm{CV}$ penetration level $=20 \mathrm{CVs}$, application data rate $=250 \mathrm{Kbps}$, and packet size $=256$ bytes . For arterials, the three speeds mentioned above represent typical speed limits.

Use Case 2: In an urban roadway condition, many CVs may communicate with the backend infrastructure through the $5 \mathrm{G}$ mmWave base stations. The number of base stations needed will depend on the penetration of CV traffic on the roadway. As such, the number of CVs is an essential factor. In use case 2 , we evaluate the performance of one the $5 \mathrm{G} \mathrm{mmWave} \mathrm{base}$ station for varying numbers of CVs $(20,40)$, along with altering the maximum speed of CVs $(35 \mathrm{mph}, 55 \mathrm{mph})$, keeping the other factors (maximum speed, application data rate, and packet size) constant. This experiment can be used to quantify the relationship between $\mathrm{CV}$ mobility and the performance of the $5 \mathrm{G} \mathrm{mmWave} \mathrm{communication.}$

Use Case 3: The primary purpose of deploying the 5G mmWave base stations is to support applications with high bandwidth requirements. However, the uplink and downlink data rates between the $\mathrm{CV}$ and the $5 \mathrm{G}$ mmWave base station will vary depending on application specifications. Moreover, the data packet size may also vary depending on the application. In use case 3, we evaluate one 5G mmWave base station's performance for variable data rate $(250 \mathrm{Kbps}, 10$ Mbps) and packet size (256 bytes, 1024 bytes), keeping the other factors (maximum speed and CV penetration level) constant. The constant values are, $\mathrm{CV}$ penetration level $=20$ $\mathrm{CVs}$, and maximum speed $=45 \mathrm{mph}$.

\section{B. Baseline Technology}

We have selected the WAVE/IEEE 802.11p standard-based communication as the baseline technology. The WAVE/IEEE $802.11 \mathrm{p}$ is an established standard for vehicular communication that uses the $5.9 \mathrm{GHz}$ spectrum, and significant research has been conducted to demonstrate its feasibility using simulations and field evaluations [28]. The federal communications commission (FCC) has recently allocated the $5.9 \mathrm{GHz}$ band to indoor $\mathrm{WiFi}$ and cellular vehicle-to-everything $(\mathrm{C}-\mathrm{V} 2 \mathrm{X})$ communication [29]. The C$\mathrm{V} 2 \mathrm{X}$ technology is based on the Release 16 of the 3GPP standard for cellular communication, and it uses the $5.9 \mathrm{GHz}$ spectrum for direct communication. The direct communication mode of $\mathrm{C}-\mathrm{V} 2 \mathrm{X}$ is called the $5 \mathrm{G}$ new radio (NR) sidelink. Although C-V2X is an emerging technology, initial studies have shown that the performance of C-V2X technology using $5.9 \mathrm{GHz}$ is very similar to the performance of WAVE/IEEE 802.11p technology [30]. That is why we have selected WAVE/IEEE 802.11p for benchmarking in this study.

\section{EXPERIMENTAL SETUP}

The details of the experimental setup for both $5 \mathrm{G}$ mmWave and WAVE/IEEE 802.11p are discussed in this section.

\section{A. 5G mmWave setup}

In this study, we have selected ns-3 as the network simulator [31] and SUMO as the microscopic-traffic simulator [32]. Using ns-3, we model the communication between the CVs and the network infrastructure the CVs is communication for the experimental setup. It is necessary to model the channel characteristics in a network device. To simulate the 5G mmWave communication in ns-3, we have used the mmWave module developed by the New York University (NYU) Wireless Group and the University of Padova [26]. The mmWave module can simulate a wide range of frequencies (i.e., $6 \sim 100 \mathrm{GHz}$ ) for $5 \mathrm{G}$ communication. The $5 \mathrm{G}$ mmWave module architecture is built upon the ns-3 LTE module (LENA), which uses the evolved packet core (EPC) network for LTE communications [33]. All layers in the 5G mmWave module from the network layer and above are identical to the LENA module. However, the mmWave PHY and MAC layers are explicitly designed for mmWave communication. We create numerous "MmWaveUeNetDevice" objects in ns-3, representing the mmWave user equipment (UE) network devices to simulate multiple CVs. We also create one "MmWaveEnbNetDevice" object in ns-3 to simulate the radio stack in the mmWave base station (evolved Node B or eNB) [26]. All parameters related to the 5G mmWave simulation modeling are given in Table I. 
TABLE I

Consideration for Wireless Communication of $\mathrm{CVs}$

\begin{tabular}{|c|c|}
\hline $\begin{array}{c}\text { Wireless } \\
\text { Communication Option }\end{array}$ & Considerations \\
\hline \multirow[t]{7}{*}{ 5G mmWave } & Frequency Band: $73 \mathrm{GHz}$ \\
\hline & Packet Size: 256 bytes, 1024 bytes \\
\hline & Data Rate: $250 \mathrm{Kbps}, 10 \mathrm{Mbps}$ \\
\hline & Blockage Model: Enabled \\
\hline & Fading Model: Small-scale Fading \\
\hline & $\begin{array}{l}\text { PHY layer: Hybrid Automatic Repeat } \\
\text { Request (HARQ) based retransmission }\end{array}$ \\
\hline & MAC Layer: Round Robin Scheduler \\
\hline \multirow[t]{7}{*}{ WAVE/IEEE $802.11 \mathrm{p}$} & Frequency Band: $5.9 \mathrm{GHz}$ \\
\hline & Packet Size: 1024 bytes \\
\hline & Data Rate: $250 \mathrm{Kbps}$ \\
\hline & Propagation Loss Model: Friis \\
\hline & Fast Fading Model: Nakagami-m \\
\hline & $\begin{array}{l}\text { Routing Protocol: Ad hoc On-Demand } \\
\text { Distance Vector (AODV) routing }\end{array}$ \\
\hline & $\begin{array}{l}\text { Transmission and Receiving Gain: } 20 \\
\mathrm{dBm}(0.1 \text { Watts })\end{array}$ \\
\hline
\end{tabular}

Later, we need to provide the CV mobility model in the simulation. The $5 \mathrm{G}$ mmWave eNB and other communication infrastructure, such as a data center working as a remote host, will have a constant position mobility model. We use SUMO to generate the trace file containing CVs' motion, such as a CV's position, speed, and timestamp of data capture. The trace file can be used to model $\mathrm{CV}$ mobility in the network simulator. The network simulation generates several output files, which are post-processed to extract the evaluation metrics such as throughput, delay, packet loss, and signal-tointerference-plus-noise ratio (SINR). The ns-3 simulation generates raw output files related to packet data convergence protocol (PDCP) and radio link control (RLC). The PDCP Tx and $\mathrm{Rx}$ files are packet traces that contain the information about each transmitted and received data packet during the simulation. The packet traces are post-processed to extract the packet information, along with timestamps and signal power. The packet information is used to generate output mapping to the evaluation metrics, which will be discussed in the following subsection. The findings presented in this paper have been obtained by running the ns- 3 simulation for a short interval of 30 seconds due to the computational requirements of the $5 \mathrm{G}$ mmWave simulations.

Our approach to communication network simulation with discrete-event network simulator (ns-3 and its predecessor ns2) has been a widely accepted strategy for designing and validating network protocols in the industry and academia for a long time [26]. The outputs generated from the ns-3 simulator have been accepted to be reliable for representing the real-world network performance [26], [34], [35]. The 5G mmWave module's channel model used in the ns-3 simulation has been validated with real-world data [36]. The details of the 5G mmWave setup, WAVE/IEEE 802.11p setup, and performance metrics are given in the following subsections.

\section{1) 5G MMWAVE CHANNEL CHARACTERISTICS}

The first part of mmWave modeling is channel characteristics modeling. We have used the NYU statistical model as the propagation path loss model in this study [26]. This propagation model uses two separate equations for path loss considering line-of-sight (LOS) and non-line-of-sight (NLOS) scenarios [26]. The communication channel matrices (i.e., beamforming vectors) are pre-generated and updated periodically (every $100 \mathrm{~ms}$ ) to reduce computation burden during simulation while considering large-scale and smallscale fading. This model also assumes blockages, as it overlays the statistical channel model with the blockage model in ns-3 and chooses the appropriate propagation path loss model. This propagation model has been calibrated for two frequencies, $28 \mathrm{GHz}$ and $73 \mathrm{GHz}$. Mobile network operators currently own the $28 \mathrm{GHz}$ spectrum, so it is not a free spectrum that can be used for vehicular communication [19].

Moreover, a higher frequency is desirable because it achieves higher throughputs and data rates, which is the focus of this study. The effect of mobility will be more severe in 73 GHz. The channel model also calculates multipath interference by using an interference computation scheme. Each multipath communication link is associated with beamforming vectors, and these vectors are used to calculate the interference. The beamforming vectors consist of several parameters, such as the departure angle and arrival angle. The error model in the mmWave module follows the LTE LENA error model [33].

\section{2) 5G MMWAVE PHY AND MAC LAYER}

Here, we describe the PHY and MAC layer properties of the mmWave module used in our simulation. The mmWave module uses a time division duplexing (TDD) frame and subframe structure following the LTE standards. However, TDD allows the module to allocate the control and data channels within the subframe in a flexible way. It also uses the hybrid automatic repeat request (HARQ) based retransmission, which helps to do fast retransmission of data packets and increases the probability of successful decoding at the data receiving end. The HARQ model is combined with channel beamforming to ensure that all transmitted packets are received. In the MAC layer, time division multiple access (TDMA) is used as the default scheme because of analog beamforming. Analog beamforming refers to the transmitter antenna arrays aligning with the receiver antenna arrays to maximize the directional gain. Analog beamforming is one type of directional transmission. The adaptive modulation and coding (AMC) mechanism is used in our simulations, which uses the channel quality indicators (CQI) to update the modulation and coding. The MAC scheduler is based on a variable transmission time interval (TTI). This study uses the round-robin scheduler, which uses the orthogonal frequency division multiplexing (OFDM) and assigns OFDM symbols to flows in a round-robin order [26].

3) THE 5G MMWAVE INTERNET STACK (TCP/IP)

The remaining layers, including the internet stack (i.e., TCP/IP protocol suite), follow the LTE LENA module [26]. The "MmWaveHelper" object in ns-3 is used to model the 5G mmWave stack in ns-3 simulation (e.g., channel, PHY, MAC). A UE (i.e., CV) is attached to the closest eNB at the 
start of the simulation. As we are interested in an end-to-end transmission scenario, a packet gateway node (PGW node) connected to the backhaul LTE core network is also created. The positions of the eNB are fixed, and the UE mobility model is derived from the trace file generated through SUMO using the "Ns2mobilityhelper" object. We have used UDP unicast transmission in this study since we want to achieve higher throughput at the cost of lower reliability.

\section{B. WAVE/IEEE 802.11p setup}

To model WAVE/IEEE $802.11 \mathrm{p}$ communication in ns-3, we have used the WAVE module developed following the IEEE 802.11p, IEEE 1609, and SAE J2735 standards. As our scenario contains multiple CVs and one roadside unit (RSU), we have followed the vehicular ad-hoc network (VANET) setup provided in ns-3 [37]. In ns-3, the focus of the WAVE/IEEE 802.11p module is on both the MAC layer and the multi-channel coordination layer. The MAC layer allows communications outside the context of a basic service set (BSS), which is known as OCB or "outside the context of a BSS." The PHY layer is like the IEEE 802.11 PHY layer. The WAVE module in ns-3 allows simulation of vehicular ad hoc network (VANET) scenarios in ns-3 to assess network performance. Specifically, it can be used to evaluate different 802.11p MAC/PHY characteristics, propagation loss models, and data routing method in realistic roadway scenarios. The WAVE example in ns 3 runs for ten simulated seconds with 40 nodes (i.e., $40 \mathrm{CVs}$ ) moving according to the random waypoint mobility model. The region size represents $300 \mathrm{mX} 1500 \mathrm{~m}$, and the CV speed is $20 \mathrm{~m} / \mathrm{s}$. The $802.11 \mathrm{p}$ communication is used with continuous access to a $10 \mathrm{MHz}$ Control Channel $(\mathrm{CH})$ for all data traffic. All nodes transmit a 200-byte basic safety messages ten times per second at 6 Mbps. The default routing protocol is AODV, and the TwoRay Ground loss model is used. The transmit power is 20 $\mathrm{dBm}$, and the transmission range is approximately $145 \mathrm{~m}$. This is similar to our case study scenario with an addition of a base station or a roadside unit (RSU). That is why we have followed this example in order to simulate the WAVE communication in this study.

The propagation loss depends on two significant factors, the distance between the communicating nodes and multipath fading. We have used the Friis propagation loss model to account for path loss due to distance and the Nakagami-m fast fading loss model to account for the path loss due to multipath fading [38]. The transmission power of $20 \mathrm{dBm}$ (equivalent to 0.1 Watts) is used in the simulation. In terms of routing protocol, we have used the ad-hoc on-demand distance vector (AODV) protocol, which is one of the widely used and most popular routing protocols for ad-hoc mobility-related scenarios [37]. The same trace files as the mmWave cases are used for node mobility. The RSU node is treated as a stationary node with a fixed position. The UDP unicast transmission mode is used to send the data packets since it offers higher maximum throughput than the UDP broadcast mode. We have used the flow monitor in ns-3, which monitors the packet flow between the communicating nodes [39]. All the relevant parameters related to simulating WAVE/IEEE $802.11 \mathrm{p}$ in ns3 are presented in Table 1.

\section{V.PERFORMANCE EVALUATION METRICS}

The details of performance evaluation metrics for both $5 \mathrm{G}$ mmWave and WAVE/IEEE $802.11 \mathrm{p}$ are discussed in this section.

\section{A. Metrics for 5G mmWave:}

The 5G mmWave module in ns-3 is configured to output the aggregate statistics of the PDCP and RLC layer for all UEs $(\mathrm{CV} s)$ and the eNodeB (eNB). The aggregate output files contain the statistics related to all data packets transmitted from and received by UEs and eNBs during the simulation using 5G mmWave communication. Since PDCP and RLC files contain similar information, we only consider the PDCP output files. Table II contains a snapshot of the data available in the PDCP output file. Here, the initial three columns represent the type of packet (transmitted or received), simulation time and ID of the cell tower or eNB, respectively. We only have one eNB in simulation, so all packets originate from cell 1. The fourth column represents RNTI (radio network temporary identifier). RNTI is assigned based on the ID assignment method in the 5G NR standard, and it is the ID of the individual CVs or UEs in simulation. The fifth and sixth column represents the packet size of the transmitted or received packet and the delay information, respectively. Only $\mathrm{RX}$ packets contain delay information: for TX packets it is empty. The evaluation metrics used in this study are packet loss, delay, throughput, and SINR. SINR is a direct output from $5 \mathrm{G}$ mmWave simulation in the RX packet trace.

Average delay: For all RX packets in Table II, there is a value on the delay column. Therefore, we filter data from the table using the ' $\mathrm{TX} / \mathrm{RX}$ ' column name for $\mathrm{RX}$ packets and take an average of the 'Delay' column to calculate the average delay for all CVs. Let us assume that the total number of RX packets is $n$ and the delay for the $i^{\text {th }}$ packet is $D_{i}$. The delay is calculated using (1).

$$
\text { Delay }=\frac{\sum_{i=1}^{n} D_{i}}{n}
$$

Throughput: Throughput is also calculated using data about simulation time and packet size, and a snapshot of the data is shown in the second column (Sim. Time) and fifth column (Packet size) of Table II. The base station or eNB continuously transmits data to the different UEs (CVs), so we consider throughput as the rate of successful data transmission from the eNB to the CVs. Let us assume that the total number of TX packets is $m$, the total number of RX packets is $n$, the simulation timestamp of transmitting the $i^{\text {th }}$ TX packet is $S t_{i}$, the simulation timestamp of receiving the $i^{\text {th }} \mathrm{RX}$ packet is $S r_{i}$ and the packet size of the $i^{\text {th }} \mathrm{RX}$ packet is $P_{i}$. Therefore, the average throughput can be calculated using (2). The average 
throughput will be less than or equal to the transmission data rate of the eNB, which is a parameter in simulation.

$$
\text { Throughput }=\frac{\sum_{i=1}^{n} P_{i} \times 8}{S r_{n}-S t_{1}}
$$

Packet Loss: Packet loss is the percentage of packets sent by the eNB that were not received by the intended UEs or CVs. Let us assume that the number of TX packets is $m$, and the number of RX packets is $n$. Therefore, packet loss is calculated using (3).

$$
\text { Packet Loss }=\frac{m-n}{m}
$$

SINR: SINR can be calculated directly from one of the output files generated by the ns-3 5G mmWave simulation, which is the RX packet trace file. In the RX packet trace, SINR is the ratio of the incoming signal's power, and the sum of the interference signal power from other objects and the noise power.

TABLE II

5G mmWave PDCP Layer Statistics from Simulation

\begin{tabular}{llllll}
\hline TX/RX & $\begin{array}{l}\text { Sim. Time } \\
(\mathrm{sec})\end{array}$ & Cell ID & RNTI & $\begin{array}{l}\text { Packet } \\
\text { (bytes) }\end{array}$ & $\begin{array}{l}\text { Dize } \\
(\mathrm{ns})\end{array}$ \\
\hline $\mathrm{Tx}$ & 0.044818 & 1 & 9 & 1054 & \\
\hline $\mathrm{Rx}$ & 0.044837 & 1 & 1 & 1054 & 863166 \\
\hline $\mathrm{Tx}$ & 0.044923 & 1 & 10 & 1054 & \\
\hline $\mathrm{Rx}$ & 0.045008 & 1 & 2 & 1054 & 928646 \\
\hline $\mathrm{Tx}$ & 0.045028 & 1 & 11 & 1054 & \\
\hline $\mathrm{Tx}$ & 0.045134 & 1 & 12 & 1054 & \\
\hline $\mathrm{Tx}$ & 0.045239 & 1 & 13 & 1054 & \\
\hline $\mathrm{Tx}$ & 0.045345 & 1 & 14 & 1054 & \\
\hline $\mathrm{Rx}$ & 0.045446 & 1 & 8 & 1054 & 733686 \\
\hline $\mathrm{Tx}$ & 0.04545 & 1 & 15 & 1054 & \\
\hline
\end{tabular}

\section{B. Metrics for Baseline Wireless Technology} (WAVE/IEEE 802.11p):

For WAVE/IEEE 802.11p scenarios (with which the 5G mmWave was compared with), we have used the flow monitor in ns-3, which monitors the packet flow between the communicating nodes. Flow monitor provides specific output values for each flow in the simulation. Here, a flow is defined as a stream of data between two unique nodes. The output measures provided by the flow monitor, which we have used in our calculation, are given below:

timeFirstTxPacket: when the first packet in a flow was transmitted

timeLastTxPacket: when the last packet in a flow was transmitted

timeFirstRxPacket: when the first packet in a flow was received by an end node

timeLastRxPacket: when the last packet in a flow was received

delaySum: sum of all end-to-end delays for all received packets of a flow

txBytes: total number of transmitted bytes for a flow

txPackets: total number of transmitted packets for a flow

rxBytes: total number of received bytes for a flow

rxPackets: total number of received packets for a flow
Based on these output values for each flow, the performance evaluation metrics are calculated using the following equations, like the $5 \mathrm{G}$ mmWave scenarios.

Average delay: The average delay for each flow can be calculated using delaySum and rxPackets as given here.

$$
\text { Delay }=\frac{\text { delaySum }}{\text { rxPackets }}
$$

Throughput: Throughput for each flow can be calculated using rxBytes, timeFirstTxPacket, and timeLastRxPacket, as given below. We convert the total bytes received to bits, and then measure the simulation time window by considering the timestamps for the first Tx packet and the last Rx packet.

\section{Throughput}

$$
=\frac{\text { rxBytes } \times 8}{\text { timeLastRxPacket }- \text { timeFirstTxPacket }}
$$

Packet Loss: Packet loss for each flow can be calculated using txPackets and rxPackets, as given below.

$$
\text { Packet Loss }=\frac{\text { txPackets }- \text { rxPackets }}{\text { txPackets }}
$$

\section{VI.EXPERIMENTAL RESULTS}

In this section, we discuss the findings based on the performance evaluation of of the $5 \mathrm{G} \mathrm{mmWave} \mathrm{and}$ WAVE/IEEE 802.11p for the different use cases in a simulation environment.

\section{A. Use Case 1}

In use case 1 , the number of CVs is 20, packet size is 1024 bytes, and transmission ( $\mathrm{Tx}$ ) bit rate (i.e., data rate) is 250 Kbps. From Figure 3, we observe that the $5 \mathrm{G}$ mmWave performance is not affected by the maximum $\mathrm{CV}$ speed for the 20 CVs. The $5 \mathrm{G}$ mmWave eNB is able to provide average throughput of 200, 199, $201 \mathrm{Kbps}$ (see Figure 3(a)), average packet loss of $21.8 \%, 22.3 \%, 21.5 \%$ (see Figure 3(b)), an average delay of 6.5, 3.8, 3.1 ms (see Figure 3(c)) for 35, 45 and $55 \mathrm{mph}$ maximum speed of CVs, respectively. Although the delay is low, the packet loss is significant, resulting in low throughput. The WAVE/IEEE 802.11p suffers from significant delay and packet loss due to the throughput requirements from multiple CVs (see Figures 3(b) and 3(d)), because WAVE/IEEE $802.11 \mathrm{p}$ has a maximum limit on the throughput for UDP unicast transmission. The RSU is able to provide average throughput of 18, 46, 14 Kbps (Figure 3(a)), average packet loss of 93.1\%, 80.9\%, 94.9\% (see Figure 3(b)) and average delay of 2.8, 2.5, 3.6 s (see Figure 3(d)) for 35, 45 and $55 \mathrm{mph}$ speed of CVs, respectively. From these results, it can be concluded that even for low data rates, the $5 \mathrm{G}$ mmWave is superior to WAVE/IEEE 802.11p in terms of packet loss, throughput, and delay. Moreover, the impact of $\mathrm{CV}$ speed on the 5G mmWave is negligible for low penetration levels of CVs in the roadway traffic stream. 


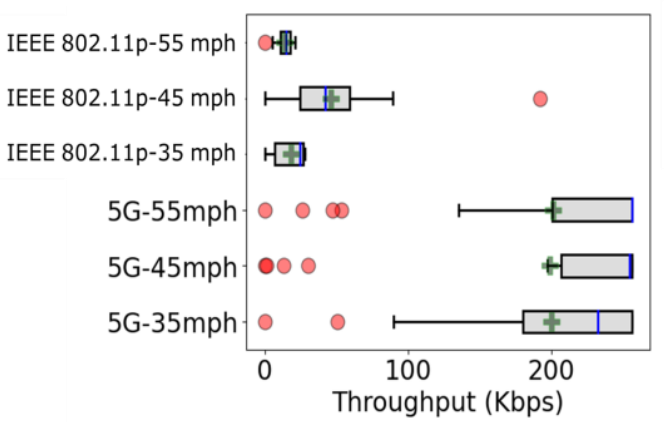

(a)

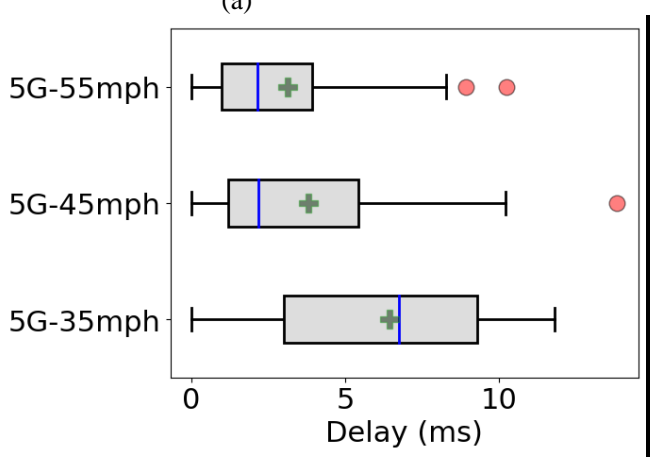

(c)

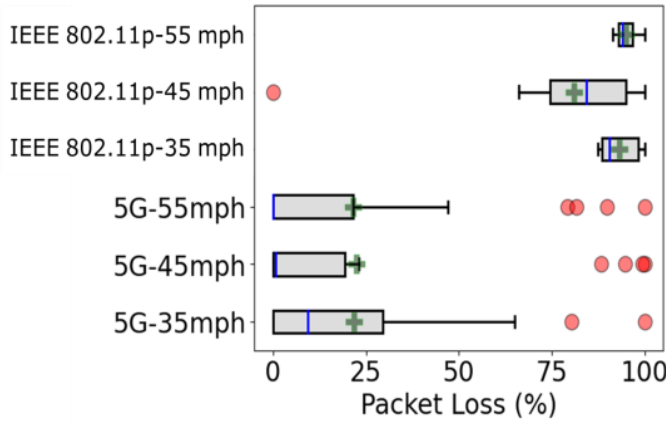

(b)

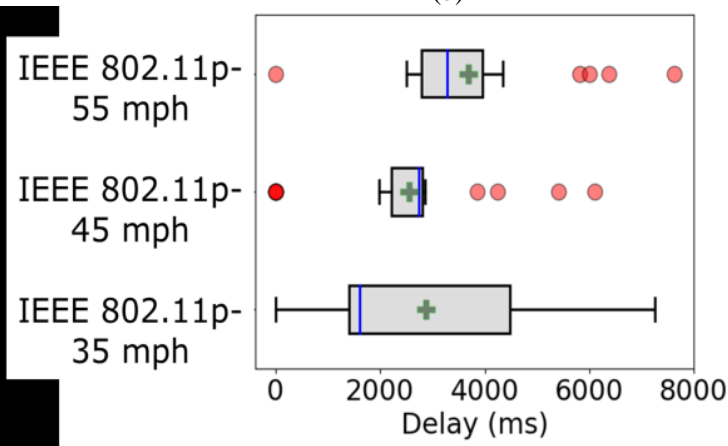

(d)

FIGURE 3. $5 \mathrm{G} \mathrm{mmWave} \mathrm{performance} \mathrm{for} \mathrm{use} \mathrm{case} 1$ and comparison with WAVE/IEEE 802.11p. (+ = Sample Mean): (a) throughput; (b) packet loss; (c) delay related to $5 \mathrm{G}$; and (d) delay related to WAVE/IEEE $802.11 \mathrm{p}$

\section{B. Use Case 2}

In use case 2, we investigate the combined effect of $\mathrm{CV}$ penetration level and $\mathrm{CV}$ mobility on communication performance. Here, we use two maximum speeds $(35 \mathrm{mph}$ and $55 \mathrm{mph}$ ) for CVs and two CV penetration levels (20 and 40 CVs). The packet size is 1024 bytes, and the Tx bit rate is 250 Kbps. From Figure 4, it can be observed that the CV penetration level has noticeable impacts on the performance of the $5 \mathrm{G}$ mmWave, regardless of the $\mathrm{CV}$ maximum speed. The increase in $\mathrm{CV}$ penetration level decreases the throughput and increases the delay and packet loss for both speeds. At $35 \mathrm{mph}$ maximum speed, the $5 \mathrm{G} \mathrm{mmWave} \mathrm{eNB}$ can provide average throughput of $200 \mathrm{Kbps}$ and $163 \mathrm{Kbps}$ (as shown in Figure 4(a)), the average delay of $11.5 \mathrm{~ms}$ and $29.8 \mathrm{~ms}$ (see Figure 4(b)), and average packet loss of $21.9 \%$ and $36.4 \%$ (see Figure 4(c)) for 20 and $40 \mathrm{CVs}$, respectively. At $55 \mathrm{mph}$ maximum speed, the $5 \mathrm{G}$ mmWave eNB can provide average throughput of 201 and $141 \mathrm{Kbps}$ (see Figure 4(a)), the average delay of

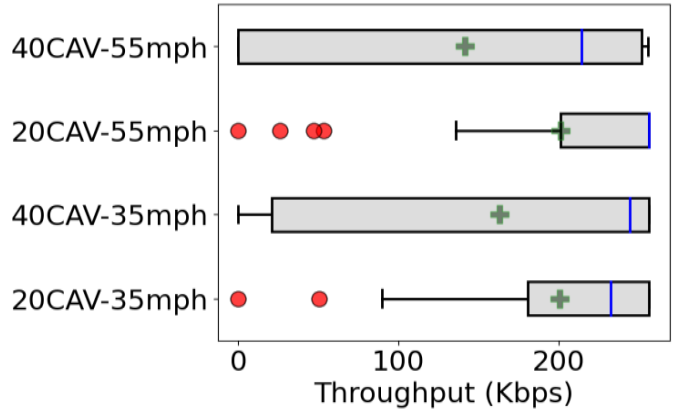

(a)

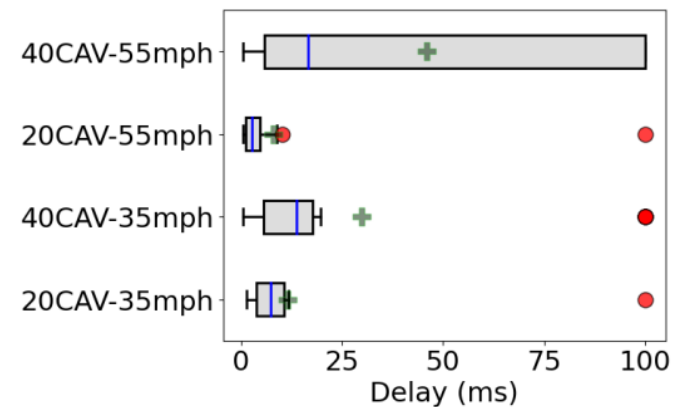

(b)

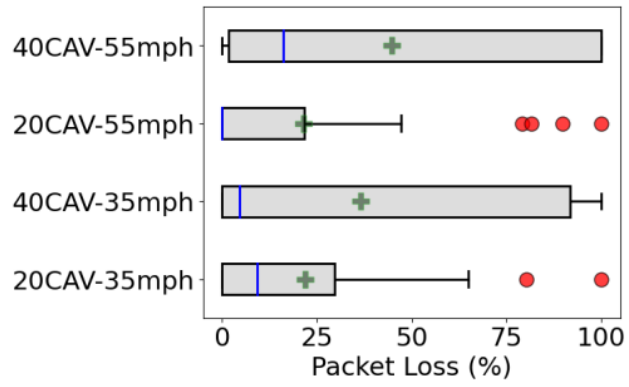

FIGURE 4. 5G mmWave performance for use case 2. (+ = Sample Mean): (a) throughput; (b) delay; and (c) packet loss 


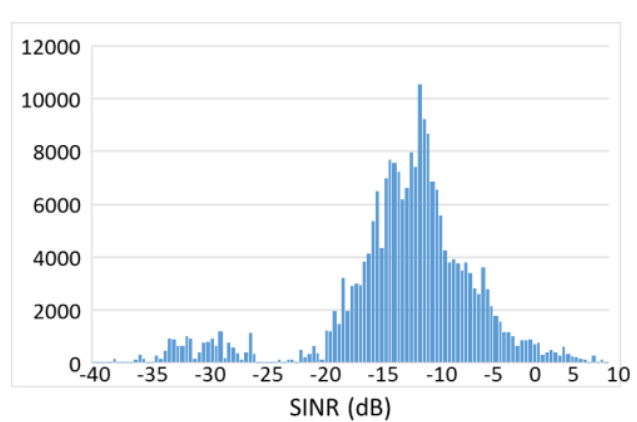

(a)

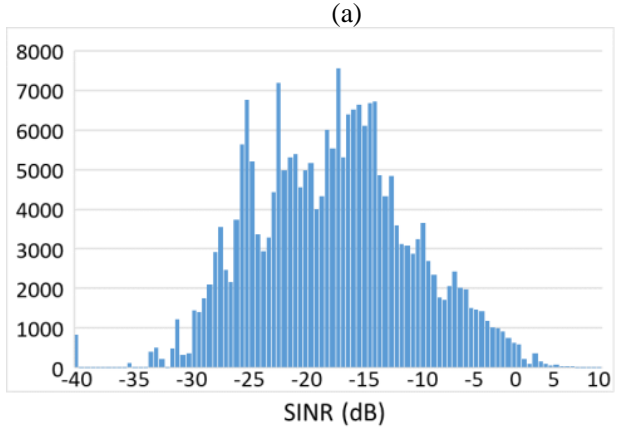

(b)

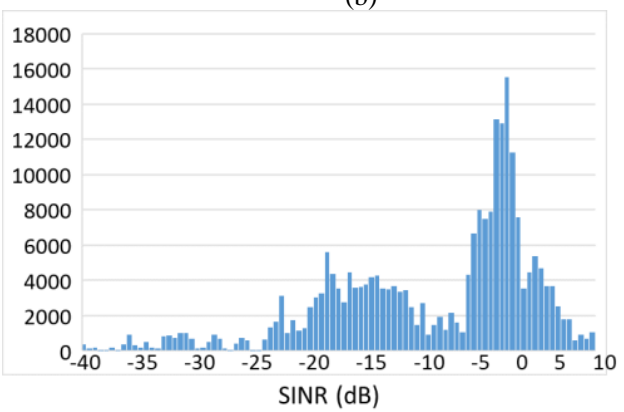

(c)

FIGURE 5. 5G mmWave SINR distribution variation with CV penetration level and V2I application data rate:

(a) $C V=20$, max speed $=45 \mathrm{mph}$, data rate $=250 \mathrm{Kbps}$;

(b) $C V=40$, max speed $=45 \mathrm{mph}$, data rate $=250 \mathrm{Kbps}$; and

(c) $C V=20$, max speed $=45 \mathrm{mph}$, data rate $=10 \mathrm{Mbps}$

8.1 and $45.8 \mathrm{~ms}$ (see Figure 4(b)), and average packet loss of $21.5 \%$ and $44.7 \%$ (see Figure 4(c)) for 20 and $40 \mathrm{CVs}$, respectively. Here, we can observe that doubling the number of CVs reduces the throughput by a factor ranging between 1.2 and 1.4, increases the average delay by factors ranging between 2.6 and 5.7, and increases packet loss by a factor ranging between 1.7 and 2.1. The increase in CVs increases the throughput requirements due to more applications downloading data using the $5 \mathrm{G}$ mmWave eNB. Moreover, more CVs in an urban area mean a higher probability of NLOS conditions [40], where any particular CV might be impeding the LOS of another CV. A scenario with a higher number of CVs also creates more multipath interference, which results in a higher loss of packets and end-to-end delays.

From the results in use case 1 (Figure 3), we have observed that the performance for $20 \mathrm{CVs}$ remains unchanged for different speeds (35 mph, $45 \mathrm{mph}, 55 \mathrm{mph}$ ). However, from Figure 4, we can observe that, for the $40 \mathrm{CV}$ scenario,

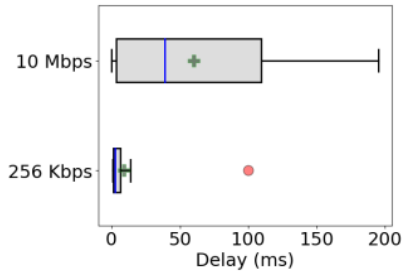

(a)

FIGURE 6. 5G mmWave performance for use case 3 (data rate). (+ = Sample Mean): (a) delay and (b) packet loss

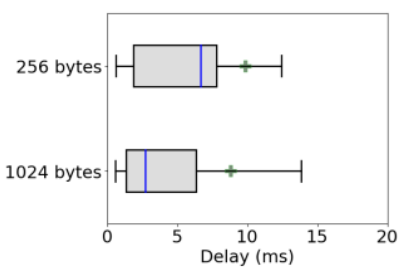

(a)

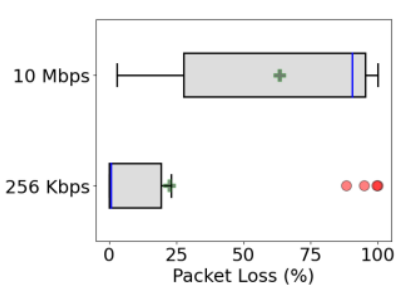

(b)

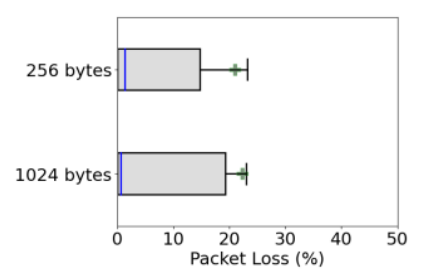

(b)

FIGURE 7. 5G mmWave performance for use case 3 (packet size) $(+=$ Sample Mean), (a) Delay, (b) Packet Loss

the $5 \mathrm{G}$ mmWave eNB performance degrades when the maximum speed is increased from $35 \mathrm{mph}$ to $55 \mathrm{mph}$. CVs' higher maximum speed also influences the $5 \mathrm{G}$ mmWave communication at higher $\mathrm{CVs}$ penetration. For $20 \mathrm{CV}$ scenarios, increasing the speed from $35 \mathrm{mph}$ to $55 \mathrm{mph}$ has no impact on the performance. However, for $40 \mathrm{CV}$ scenarios, increasing the speed from 35 to $55 \mathrm{mph}$ decreases the average throughput by a factor of 1.2 and increases the delay and packet loss by factors of 1.5 and 1.2, respectively. This is an interesting finding from use case 2 , as it shows that the challenge factors are not independent. Instead, they can influence each other in affecting communication performance.

We have also measured the quality of the $5 \mathrm{G}$ mmWave communication in terms of SINR. SINR is a wireless quality indicator measured in decibels $(\mathrm{dB})$. In a frequency distribution plot of SINR, a higher frequency at higher $\mathrm{dB}$ values represent better quality of wireless communication with higher throughput. Figures 5(a) and 5(b) show that interference and noise increase with the higher number of CVs. For $\mathrm{CV}=20$, the SINR is mostly between $-20 \mathrm{~dB}$ and 0 $\mathrm{dB}$, but for $\mathrm{CV}=40$, the SINR is mostly spread out between 35 $\mathrm{dB}$ and $0 \mathrm{~dB}$.

\section{Use Case 3}

In use case 3, we examine the effect of V2I application specifications, specifically data rate and packet size, on the network performance of the $5 \mathrm{G}$ mmWave. At first, we investigated the effect of higher data rates on the performance of the $5 \mathrm{G}$ mmWave. Thus, we increase the data rate from 250 Kbps to $10 \mathrm{Mbps}$ for each $\mathrm{CV}$, keeping the packet size fixed at 1024 bytes. In this case, we use $20 \mathrm{CV}$ s at an average speed of $45 \mathrm{mph}$. From Figure 6, we observe that a higher bit rate has a noticeable impact on network performance. The average throughput is higher because of the higher Tx bit rate. We have found that for the $10 \mathrm{Mbps}$ case, the average delay is $59.9 \mathrm{~ms}$ compared to the $8.8 \mathrm{~ms}$ for the $250 \mathrm{Kbps}$ case, so the delay increases by a factor of 6.8 when the bit rate increases by a 
factor of 40 (Figure 6(a)). The average packet loss increases from $22.3 \%$ (for the $250 \mathrm{Kbps}$ case) to $63.3 \%$ (for the $10 \mathrm{Mbps}$ case), which is an increase by a factor of 2.8 (Figure 6(b)).

We examine the effect of packet size on the network performance of the $5 \mathrm{G}$ mmWave. We decrease the packet size from 1024 bytes to 256 bytes for each CV, keeping the data rate fixed at $250 \mathrm{Kbps}$. For this case, we use $20 \mathrm{CVs}$ at an average speed of $45 \mathrm{mph}$. From Figure 7, we observe that a change in packet size has no significant impact on the delay (Figure 7(a)) and packet loss (Figure 7(b)). The average delay is $9.8 \mathrm{~ms}$ compared to the $8.8 \mathrm{~ms}$ for the 1024 bytes case, and the average packet loss is $21 \%$ compared to $22.3 \%$ for the 1024 bytes case.

As shown in Figure 5(c), the SINR distribution is improved compared to Figures 5(a) and 5(b) due to the higher Tx bit rate and higher throughput. Throughput is logarithmically proportional to SINR, as defined by the Shannon-Hartley theorem [41].

\section{CONCLUSIONS}

Based on the result presented in Section VI, the CV penetration level, mobility, and the application data rate have noticeable impacts on the performance of the $5 \mathrm{G}$ mmWave. Our analysis has revealed that doubling the CV penetration level reduces the throughput by a factor ranging between 1.2 and 1.4, increases the average delay by factors ranging between 2.6 and 5.7, and increases packet loss by a factor ranging between 1.7 and 2.1. Moreover, for a higher CV penetration level, CV speed has an impact on network performance. However, this impact is not present for the lower $\mathrm{CV}$ penetration level. The $\mathrm{CV}$ application data rate also has a significant effect on the performance. For an increase in data rate by a factor of 40 , the average delay increases by a factor of 6.8 , whereas the average packet loss increases by a factor of 2.8. Changing the packet size did not have any impact on the delay, packet loss, and throughput. Also, we found higher noise and interference when the $\mathrm{CV}$ penetration level increases and the data rate increases, which leads to the degradation of network performance. Through these findings, we have identified that one $5 \mathrm{G}$ mmWave base station operating at a very high frequency $(73 \mathrm{GHz})$ might be insufficient to serve the CVs. The potential solutions for supporting CV applications may include deploying multiple $5 \mathrm{G}$ mmWave base stations or leveraging existing $5 \mathrm{G}$ base stations.

\section{VIII.RECOMMENDATIONS FOR FUTURE RESEARCH}

Our recommendations for future research are divided into two subsections: simulation and field evaluations.

\section{A. Simulation-based research}

This study does not consider the effects of buildings and blockages on the $5 \mathrm{G}$ mmWave communication. It only shows the $5 \mathrm{G}$ mmWave's performance in the LOS scenario. The $5 \mathrm{G}$ mmWave signals are susceptible to signal blockage and
NLOS. Future research should focus on simulating different types of scenarios considering buildings and blockages (e.g., urban roadway tunnels, rural areas) to investigate its effect on the performance of the $5 \mathrm{G}$ mmWave.

In this study, we have simulated a limited number of CVs on the road. Future studies should investigate a larger area with a higher number of CVs. These future case studies can identify the shortcomings in $5 \mathrm{G}$ mmWave communication with a higher penetration of $\mathrm{CVs}$ in urban network and could point towards further improvements in the $5 \mathrm{G} \mathrm{mmWave}$ technology. We have simulated a portion of a corridor $(\sim 500 \mathrm{~m})$ and the performance of one $5 \mathrm{G}$ mmWave base station for this particular area. Future research should investigate multiple $5 \mathrm{G}$ mmWave base stations' performance, different spacing between the base stations and intelligent handoff management from $\mathrm{CVs}$ to perform horizontal handover between the base stations. A heterogeneous network with the 5G mmWave and other communication technology (i.e., 4G, LTE, DSRC, WiFi) provides a reliable communication system for an extended travel area, such as intercity highways. CVs will perform vertical handover between communication technologies based on their availability and coverage.

\section{B. Field evaluation}

A part of the $5 \mathrm{G}$ rollout nationally includes mmWave base station deployments, especially in urban areas with high user density. Therefore, along with detailed sensitivity analysis through simulations, the $5 \mathrm{G}$ mmWave should also be evaluated using field tests in urban areas containing many users. The evaluation may include the validation of simulation results and updating simulation parameters. A connected vehicle testbed containing the $5 \mathrm{G}$ mmWave base stations will be ideal for conducting further research and evaluating communication performance. Finally, we should develop testbeds having the $5 \mathrm{G} \mathrm{mmWave} \mathrm{base} \mathrm{stations} \mathrm{to} \mathrm{test} \mathrm{different}$ $\mathrm{CV}$ (V2V and V2I) applications.

\section{ACKNOWLEDGMENT}

This study is supported by the Center for Connected Multimodal Mobility $\left(\mathrm{C}^{2} \mathrm{M}^{2}\right)$ (a US Department of Transportation Tier 1 University Transportation Center) headquartered at Clemson University, Clemson, South Carolina, USA. Any opinions, findings, conclusions, and recommendations expressed in this material are those of the author(s). They do not necessarily reflect the views of $\mathrm{C}^{2} \mathrm{M}^{2}$, and the US Government assumes no liability for the contents or use thereof.

\section{REFERENCES}

[1] USDOT, “Architecture Reference for Cooperative and Intelligent Transportation,” 2021. https://local.iteris.com/arc-it/ (accessed Apr. 14, 2021).

[2] D. Roy, M. Chatterjee, and E. Pasiliao, "Video quality assessment for inter-vehicular streaming with IEEE 802.11p, LTE, and LTE Direct networks over fading channels," Computer Communications, vol. 118, pp. 69-80, Mar. 2018, doi: 10.1016/j.comcom.2017.09.010. 
[3] T. S. Rappaport, G. R. MacCartney, S. Sun, H. Yan, and S. Deng, "Small-Scale, Local Area, and Transitional Millimeter Wave Propagation for 5G Communications," IEEE Transactions on Antennas and Propagation, vol. 65, no. 12, pp. 6474-6490, Dec. 2017, doi: 10.1109/TAP.2017.2734159.

[4] I. Mavromatis, A. Tassi, R. J. Piechocki, and A. Nix, "Efficient V2V Communication Scheme for 5G mmWave Hyper-Connected CAVs," in 2018 IEEE International Conference on Communications Workshops (ICC Workshops), May 2018, pp. 1-6. doi: 10.1109/ICCW.2018.8403780. [5] I. Rasheed and F. Hu, "Intelligent super-fast Vehicle-to-Everything $5 \mathrm{G}$ communications with predictive switching between mmWave and $\mathrm{THz}$ links," Vehicular Communications, vol. 27, p. 100303, Jan. 2021, doi: 10.1016/j.vehcom.2020.100303.

[6] Y. Li, C.-Y.-D. Sim, Y. Luo, and G. Yang, "12-Port 5G Massive MIMO Antenna Array in Sub-6GHz Mobile Handset for LTE Bands 42/43/46 Applications," IEEE Access, vol. 6, pp. 344-354, 2018, doi: 10.1109/ACCESS.2017.2763161.

[7] P. Adhikari, "Understanding Millimeter Wave Wireless Communication," undefined, 2008, Accessed: Sep. 09, 2021. [Online]. Available: https://www.semanticscholar.org/paper/UnderstandingMillimeter-Wave-Wireless-

Adhikari/540ad9ad8412733dd235715be44f978434164da9

[8] M. Xiao et al., "Millimeter Wave Communications for Future Mobile Networks," IEEE Journal on Selected Areas in Communications, vol. 35, no. 9, pp. 1909-1935, Sep. 2017, doi: 10.1109/JSAC.2017.2719924.

[9] I. Rasheed, F. Hu, Y.-K. Hong, and B. Balasubramanian, "Intelligent Vehicle Network Routing With Adaptive 3D Beam Alignment for mmWave 5G-Based V2X Communications," IEEE Transactions on Intelligent Transportation Systems, vol. 22, no. 5, pp. 2706-2718, May 2021, doi: 10.1109/TITS.2020.2973859.

[10]C. R. Storck and F. Duarte-Figueiredo, "A 5G V2X Ecosystem Providing Internet of Vehicles," Sensors, vol. 19, no. 3, Art. no. 3, Jan. 2019, doi: 10.3390/s19030550.

[11] J. Hu, C. Chen, T. Qiu, and Q. Pei, "Regional-Centralized Content Dissemination for eV2X Services in 5G mmWave-Enabled IoV," IEEE Internet of Things Journal, vol. 7, no. 8, pp. 7234-7249, Aug. 2020, doi: 10.1109/JIOT.2020.2982983.

[12] R. He et al., "Propagation Channels of 5G Millimeter-Wave Vehicleto-Vehicle Communications: Recent Advances and Future Challenges," IEEE Vehicular Technology Magazine, vol. 15, no. 1, pp. 16-26, Mar. 2020, doi: 10.1109/MVT.2019.2928898.

[13] A. Bohli and R. Bouallegue, "How to Meet Increased Capacities by Future Green 5G Networks: A Survey," IEEE Access, vol. 7, pp. 4222042237, 2019, doi: 10.1109/ACCESS.2019.2907284.

[14] K. C. Dey, A. Rayamajhi, M. Chowdhury, P. Bhavsar, and J. Martin, "Vehicle-to-vehicle (V2V) and vehicle-to-infrastructure (V2I) communication in a heterogeneous wireless network - Performance evaluation," Transportation Research Part C: Emerging Technologies, vol. 68, pp. 168-184, Jul. 2016, doi: 10.1016/j.trc.2016.03.008.

[15] A. S. Huang et al., "A High-rate, Heterogeneous Data Set From The DARPA Urban Challenge," The International Journal of Robotics Research, vol. 29, no. 13, pp. 1595-1601, Nov. 2010, doi: $10.1177 / 0278364910384295$.

[16]D. Brake, "A U.S. National Strategy for 5G and Future Wireless Innovation," Information Technology and Innovation Foundation, Apr. 2020. Accessed: Sep. 09, 2021. [Online]. Available:

https://itif.org/publications/2020/04/27/us-national-strategy-5g-and-futurewireless-innovation

[17] P. Datta and S. Kaushal, "Exploration and comparison of different 4G technologies implementations: A survey," in 2014 Recent Advances in Engineering and Computational Sciences (RAECS), Mar. 2014, pp. 1-6. doi: 10.1109/RAECS.2014.6799517

[18] S. Ahmadi, 5G NR - Architecture, Technology, Implementation, and Operation of 3GPP New Radio Standards, 1st ed. Academic Press, 2019. [19]D. Garcia-Roger, E. E. González, D. Martín-Sacristán, and J. F. Monserrat, "V2X Support in 3GPP Specifications: From 4G to 5G and Beyond," IEEE Access, vol. 8, pp. 190946-190963, 2020, doi: 10.1109/ACCESS.2020.3028621.

[20] N. Loulloudes, G. Pallis, and M. D. Dikaiakos, "The Dynamics of Vehicular Networks in Urban Environments," arXiv:1007.4106 [cs], Oct. 2011, Accessed: Sep. 09, 2021. [Online]. Available: http://arxiv.org/abs/1007.4106
[21] M. Sepulcre, J. Mittag, P. Santi, H. Hartenstein, and J. Gozalvez, "Congestion and Awareness Control in Cooperative Vehicular Systems," Proceedings of the IEEE, vol. 99, no. 7, pp. 1260-1279, Jul. 2011, doi: 10.1109/JPROC.2011.2116751

[22] R. Stanica, E. Chaput, and A.-L. Beylot, "Local density estimation for contention window adaptation in vehicular networks," in 2011 IEEE 22nd International Symposium on Personal, Indoor and Mobile Radio Communications, Sep. 2011, pp. 730-734. doi:

10.1109/PIMRC.2011.6140062.

[23] M. Afaq, J. Iqbal, T. Ahmed, I. Ul Islam, M. Khan, and M. S. Khan, "Towards 5G network slicing for vehicular ad-hoc networks: An end-toend approach," Computer Communications, vol. 149, pp. 252-258, Jan. 2020, doi: 10.1016/j.comcom.2019.10.018.

[24] C. Dehos, J. L. González, A. De Domenico, D. Kténas, and L. Dussopt, "Millimeter-wave access and backhauling: the solution to the exponential data traffic increase in $5 \mathrm{G}$ mobile communications systems?," IEEE Communications Magazine, vol. 52, no. 9, pp. 88-95, Sep. 2014, doi: 10.1109/MCOM.2014.6894457.

[25] A. Mastrosimone and D. Panno, "A comparative analysis of mmWave vs LTE technology for 5G Moving Networks," in 2015 IEEE 11th International Conference on Wireless and Mobile Computing, Networking and Communications (WiMob), Oct. 2015, pp. 422-429. doi: 10.1109/WiMOB.2015.7347993.

[26] M. Mezzavilla et al., "End-to-End Simulation of $5 \mathrm{G}$ mmWave Networks," IEEE Communications Surveys Tutorials, vol. 20, no. 3, pp. 2237-2263, 2018, doi: 10.1109/COMST.2018.2828880.

[27] M. Giordani, A. Zanella, and M. Zorzi, "Technical Report MillimeterWave Communication in Vehicular Networks: Coverage and Connectivity Analysis," arXiv:1705.06960 [cs, math], Apr. 2017, Accessed: Oct. 27, 2021. [Online]. Available: http://arxiv.org/abs/1705.06960

[28]F. Arena, G. Pau, and A. Severino, "A Review on IEEE 802.11p for Intelligent Transportation Systems," Journal of Sensor and Actuator Networks, vol. 9, no. 2, Art. no. 2, Jun. 2020, doi: 10.3390/jsan9020022. [29] SAE, "Vehicle safety communications landscape clarifies with controversial FCC ruling," 2021.

https://www.sae.org/site/news/2020/11/fcc-5.9-ghz-cv2x-decision (accessed Sep. 10, 2021).

[30] V. Mannoni, V. Berg, S. Sesia, and E. Perraud, "A Comparison of the V2X Communication Systems: ITS-G5 and C-V2X," in 2019 IEEE 89th Vehicular Technology Conference (VTC2019-Spring), Apr. 2019, pp. 1-5. doi: 10.1109/VTCSpring.2019.8746562.

[31] G. Carneiro, "NS-3: Network simulator 3.," 2010

https://www.nsnam.org/tutorials/NS-3-LABMEETING-1.pdf

[32] M. Behrisch, L. Bieker, J. Erdmann, and D. Krajzewicz, "SUMO Simulation of Urban MObility: An overview," in in SIMUL 2011, The Third International Conference on Advances in System Simulation, 2011, pp. 63-68.

[33] N. Baldo, "The ns-3 LTE module by the LENA project," 2011. https://www2.nsnam.org/tutorials/tutorials/consortium13/lte-tutorial.pdf [34]F. Van den Abeele, J. Haxhibeqiri, I. Moerman, and J. Hoebeke, "Scalability Analysis of Large-Scale LoRaWAN Networks in ns-3," IEEE Internet of Things Journal, vol. 4, no. 6, pp. 2186-2198, Dec. 2017, doi: 10.1109/JIOT.2017.2768498

[35]Z. Hossain, Q. Xia, and J. M. Jornet, "TeraSim: An ns-3 extension to simulate Terahertz-band communication networks," Nano Communication Networks, vol. 17, pp. 36-44, Sep. 2018, doi:

10.1016/j.nancom.2018.08.001

[36] M. R. Akdeniz et al., "Millimeter Wave Channel Modeling and Cellular Capacity Evaluation," IEEE Journal on Selected Areas in Communications, vol. 32, no. 6, pp. 1164-1179, Jun. 2014, doi: 10.1109/JSAC.2014.2328154.

[37] R. Chitraxi, U. Upadhayaya, T. Makwana, and P. Mahida, "Simulation of VANET using ns-3 and SUMO," International Journal of Advanced Research in Computer Science and Software Engineering, vol. 4, no. 4, pp. 563-569, 2014

[38]Z. H. Mir and F. Filali, "On the Performance Comparison between IEEE 802.11p and LTE-Based Vehicular Networks," in 2014 IEEE 79th Vehicular Technology Conference (VTC Spring), May 2014, pp. 1-5. doi: 10.1109/VTCSpring.2014.7023017.

[39] G. Carneiro, P. Fortuna, and M. Ricardo, "FlowMonitor: a network monitoring framework for the network simulator 3 (NS-3)," in 
Proceedings of the Fourth International ICST Conference on Performance Evaluation Methodologies and Tools, Brussels, BEL, Oct. 2009, pp. 1-10. doi: 10.4108/ICST.VALUETOOLS2009.7493.

[40] L. Montero, C. Ballesteros, C. de Marco, and L. Jofre, "Beam management for vehicle-to-vehicle (V2V) communications in millimeter wave 5G," Vehicular Communications, p. 100424, Oct. 2021, doi: 10.1016/j.vehcom.2021.100424

[41]C. E. Shannon, "A mathematical theory of communication," SIGMOBILE Mob. Comput. Commun. Rev., vol. 5, no. 1, pp. 3-55, Jan. 2001, doi: 10.1145/584091.584093.

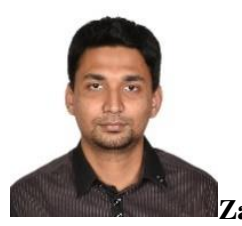
Glenn Department of Civil Engineering at Clemson University, and the B.Sc. degree in electrical and electronic engineering from Bangladesh University of Engineering and Technology (BUET), Dhaka, Bangladesh, in 2014. Then he served as a petroleum engineer in asset development department of Chevron BPC (Bangladesh Profit Center) from 2014 to 2016. $\mathrm{He}$ is currently working as the Senior Research Scientist at Walmart. His primary research focus is connected and autonomous vehicles (CAVs). Within the CAV domain, his research interests are machine/deep learning, computer networking (V2X, SDN, HetNet), data analytics (data fusion, big data), cloud computing and cybersecurity.

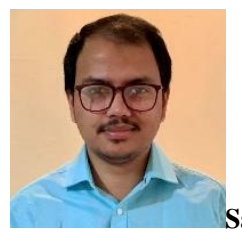

Sakib Mahmud Khan (Member'20) is the Assistant Research Professor at Glenn Department of Civil Engineering, Clemson University, and Assistant Director of the Center for Connected Multimodal Mobility $\left(\mathrm{C}^{2} \mathrm{M}^{2}\right)$. Before joining the center, he was a post-doctoral research scholar working at California Partners for Advanced Transportation Technology (PATH), University of California Berkeley. He received his $\mathrm{Ph}$.D. and M.Sc. in Civil Engineering from Clemson University in 2019 and 2015, respectively.

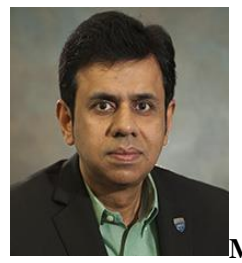

Mashrur Chowdhury (SM'12) received the Ph.D. degree in civil engineering from the University of Virginia, USA in 1995. Prior to entering academia in August 2000, he was a Senior ITS Systems Engineer with Iteris Inc. and a Senior Engineer with Bellomo-McGee Inc., where he served as a Consultant to many state and local agencies, and the U.S. Department of Transportation on ITS related projects. He is the Eugene Douglas Mays Professor of Transportation with the Glenn Department of Civil Engineering, Clemson University, SC, USA. He is also a Professor of Automotive Engineering and a Professor of Computer Science at Clemson University. $\mathrm{He}$ is the Director of the USDOT Center for Connected Multimodal Mobility (a TIER 1 USDOT University Transportation Center). $\mathrm{He}$ is Co-Director of the Complex Systems, Data Analytics and Visualization Institute (CSAVI) at Clemson University. Dr. Chowdhury is the Roadway-Traffic Group lead in the Connected Vehicle Technology Consortium at Clemson University. $\mathrm{He}$ is also the Director of the Transportation Cyber-Physical Systems Laboratory at Clemson University. Dr. Chowdhury is a Registered Professional Engineer in Ohio, USA. He serves as an Associate Editor for the IEEE TRANSACTIONS ON
INTELLIGENT TRANSPORTATION SYSTEMS. He is a Fellow of the American Society of Civil Engineers and a Senior Member of IEEE.

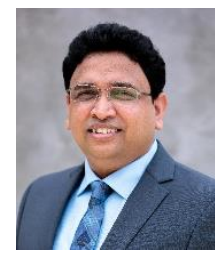

Mizanur Rahman is an assistant professor in the Department of Civil, Construction and Environmental Engineering at the University of Alabama, Tuscaloosa, Alabama. He received his M.Sc. and $\mathrm{Ph} . D$. degrees in Civil Engineering (Transportation systems), from Clemson University, in 2013 and 2018, respectively. After his graduation in August 2018, he joined as a postdoctoral research fellow for the Center for Connected Multimodal Mobility $\left(\mathrm{C}^{2} \mathrm{M}^{2}\right)$, a U.S. Department of Transportation Tier 1 University Transportation Center (cecas.clenson.edu/c2m2). After that, he has also served as an Assistant Director of C2M2. He was closely involved in the development of South Carolina Connected Vehicle Testbed (SC-CVT). His research focuses on traffic flow theory, and transportation cyber-physical systems for connected and automated vehicles and smart cities.

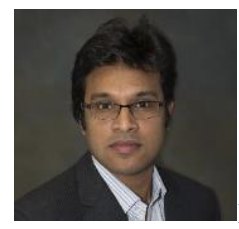

Mhafuzul Islam received the BS degree in Computer Science and Engineering from the Bangladesh University of Engineering and Technology (BUET) in 2014, MS and Ph.D. degree in Civil Engineering from Clemson University in 2018 and 2021, respectively. He is currently working as a Senior Research Scientist in General Motors. His research interests include Transportation Cyber-Physical Systems with an emphasis on Data-driven Connected and Autonomous Vehicle. 